\title{
Analysis and Prediction for the Voltage Spike of Power Cable based on the Regression Model
}

\author{
Wei Xia, Yongbin Bai, Fenghai Ding, Bin Qiu, Shenxiang Gao
}

China Satellite Maritime Tracking and Control Department, Jiangyin 214431, China

Keywords: voltage spike, regression model, variance analysis, F-test.

\begin{abstract}
In view of the problem that the test of spike signal in the power line is difficult to capture, and based on the theory of time - domain analysis of dynamic circuit, the influence factors of voltage spike test by using the maximum value of traditional multi - random test are studied. By means of the statistical analysis of the test data, a voltage spike prediction method based on linear regression model is proposed. According to the principle of correlation test of variance analysis, the validity of the regression model is verified. The accuracy of the prediction results is evaluated.
\end{abstract}

\section{Introduction}

With the continuous development of power supply technology and users of the continuous improvement of the quality requirements of electricity, voltage surge, spikes and other interference has gradually become a guarantee for the quality of the user safety and uninterrupted power supply to consider the main issues. Wherein the power line conduction interference associated with the electrical device switch is a multiple event in the power system which is susceptible to misuse or damage to the automation equipment based on microelectronics and computer technology. However, in the specific implementation process, since the formation of the above-mentioned interference signal is related to the voltage instantaneous value of the switching operation, the random switch test is often difficult to traverse all the states, resulting in the problem that the maximum peak value of the interference signal is difficult to capture.

\section{Principle of power line voltage spike test}

To GJB152A CE107 voltage spike signal transmission test, for example, set the test equipment (EUT) for the inductive load, the test principle shown in Figure 1 below.

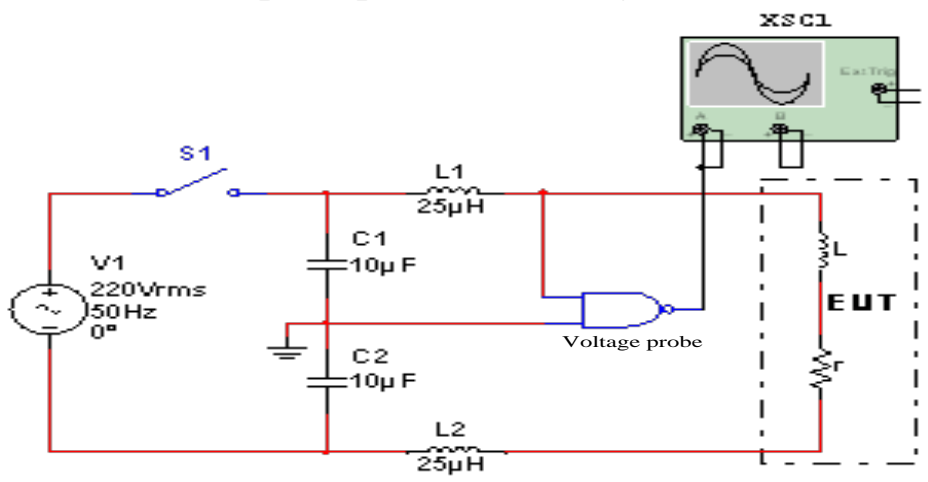

Figure 1. Power line spike signal open time domain test configuration diagram

The main test steps are as follows:

(1). As shown in Figure 1, the measurement device layout, in which $25 \mathrm{uH}$ inductance and $10 \mathrm{uF}$ capacitor together to form a test coupling network, in the $10 \mathrm{kHz}$ to $50 \mathrm{MHz}$ frequency band to form a band-pass filter, the output of the voltage probe connected to the memory oscilloscope or peak voltage meter;

(2). The EUT of the test equipment is subjected to multiple switching operations to measure the maximum peak value of the spike signal and record it. 
The following analysis of the test process, when the switch $S$ is off, the reference dynamic circuit time domain analysis theory, according to the three elements of the formula, test coupling voltage spikes can be expressed by the following approximate formula.

$$
\mu_{R}(t) \cong-\frac{R}{2} I_{L 0} \sin \left(\omega t+\theta_{0}\right) e^{\frac{-t}{\tau}}
$$

In the actual experiment, it can be seen that the probability of catching the voltage spike through a limited number of switching tests is very low, and the result of the test is usually smaller than the real result, which is an unfavorable factor for the evaluation of EMC safety margin. Therefore, how to predict the peak voltage by using the finite number of random test results to optimize the experimental scheme is the problem to be solved in this paper.

\section{Design of Voltage Spike Test Scheme}

In order to solve the above problems, it is necessary to analyze the factors that affect the voltage peak. For the circuit shown in Fig. 1, when the switch is turned off, the influencing factors of the voltage peak include the off-load transient current, the impedance parameters of the tested equipment, Test the coupling network parameters (including the impedance parameters of the oscilloscope voltage probe). For the experimental configuration has been identified, the latter two are fixed, this time the main factors for the instantaneous current, indicating that the peak voltage can be estimated by the instantaneous current. However, due to the above test configuration can not give the instantaneous current, it is necessary in Figure 1 test loop to add a current probe, used to measure the instantaneous current when the switch is off, but the current probe will lead to the voltage peak attenuation It is not clear, so you can pass through the current loop number of turns as a factor to verify whether it will have an impact on the voltage peak. The final test configuration is shown in Figure 2 below. The test equipment model information is shown in Table 1 below.

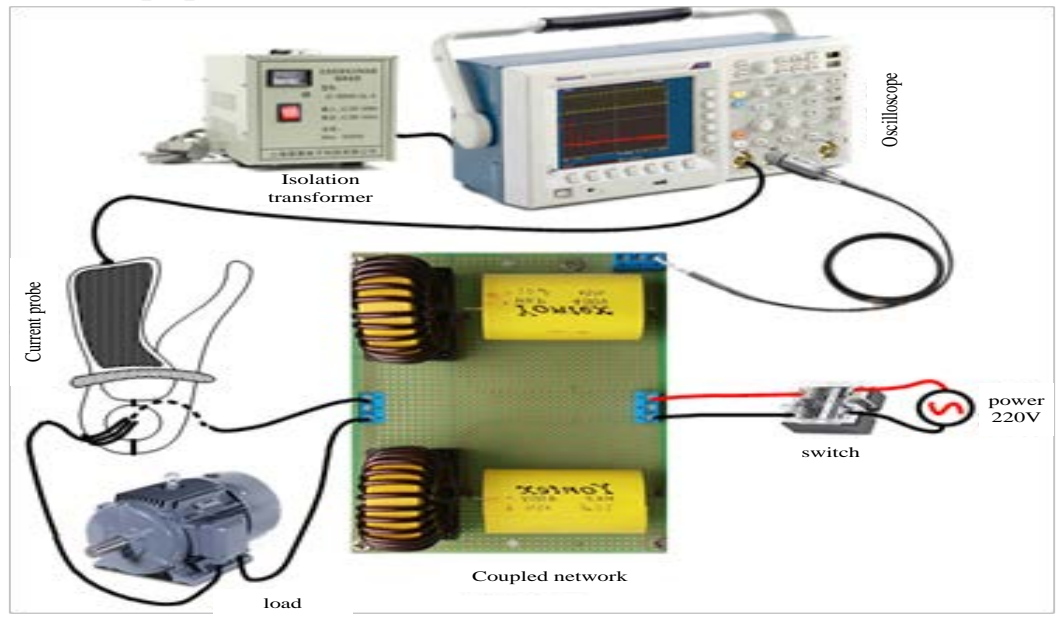

Figure 2. Power line voltage spike test configuration design

Table 1. Test equipment model information table

\begin{tabular}{cccc}
\hline number & types & name & Model parameters \\
\hline 1 & & Digital oscilloscope & TDS3052B \\
2 & & Voltage probe & Tektronix P5100 \\
3 & Testing equipment & Current probe & Agilent N2780B \\
4 & & Coupling capacitance & 941C12W1K-F \\
5 & & Coupling inductance & 769914 \\
\hline 6 & Tested equipment & Induction motor & YY6324 \\
\hline
\end{tabular}

According to the test equipment configuration shown in Fig. 2 and Table 1, the test system is established, and the voltage spike and instantaneous current when the switch is off are measured as shown in Fig. 3 and 4, in which the wire passing through the current probe is 12 turns. 


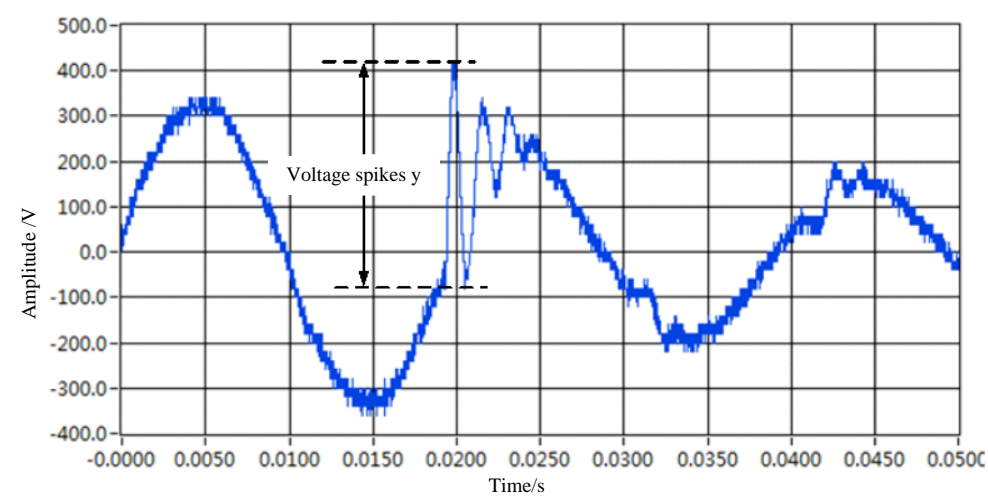

Figure 3. Voltage spike waveform

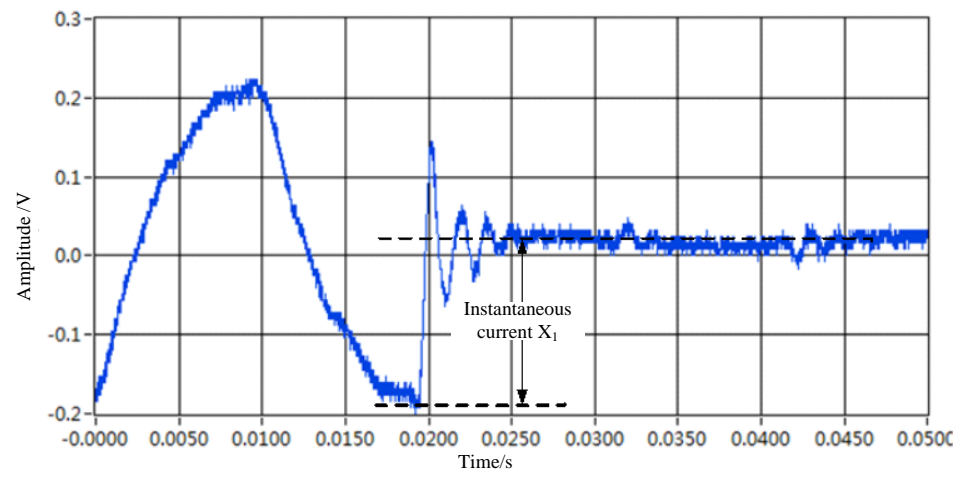

Figure 4. Instantaneous current waveform (conduction factor 0.01V / A)

\section{Voltage spike prediction based on linear regression model}

The following analysis of the data of the test results, including the following aspects, the least squares estimation, test the linear equation of the significant and determine the factors of the order, when the regression equation and the regression coefficient is significant, the voltage spike to predict And evaluation.

\subsection{Correlation test based on variance analysis - $\mathbf{F}$ test}

For a set of data of any two variables $\mathrm{x}$ and $\mathrm{y}$, the regression equation can be fitted by the least squares method, but whether the fitting regression equation conforms to the objective laws between the variables $\mathrm{x}$ and $\mathrm{y}$ needs to be verified by the regression equation To solve. The variance between $\mathrm{n}$ observations can be expressed as the sum of squares of the observed values and their arithmetic mean values, denoted by:

$$
S S T=\sum_{i=1}^{n}\left(y_{i}-\bar{y}\right)^{2}
$$

It can be broken down into two parts:

$$
\begin{gathered}
S S R=\sum_{i=1}^{n}\left(\hat{y}_{i}-\bar{y}\right)^{2} \\
S S E=\sum_{i=1}^{n}\left(y_{i}-\hat{y}_{i}\right)^{2}
\end{gathered}
$$

Where SSR is called the sum of squares of regression, which reflects the change in the total variation of the output quantity y due to the different values of the independent variable $\mathrm{x}$; the SSE is called the residual square sum, that is, the residual error of all the observed values from the regression line Square sum, which is the variation effect of $\mathrm{y}$ other than the linear effect of $\mathrm{x}$ on $\mathrm{y}$ (including the experimental error, the non-linear effect of $\mathrm{x}$ on $\mathrm{y}$, and other uncontrolled factors). Corresponding to the decomposition of SST, the degree of freedom also has a corresponding decomposition.

To test whether there is a significant linear relationship between equation (3), the following assumptions can be examined:

$$
\left\{\begin{array}{c}
H_{0}: b_{1}=b_{2}=0 \\
H_{1}: b_{1} \text { and } b_{2} \text { at least one is not zero }
\end{array}\right.
$$


According to the F test method, construct the following test statistic:

$$
F \widehat{=} \frac{M S R}{M S E}
$$

The above formula shows that this linear regression relationship is highly significant. The regression relationship does not mean that the effect of each independent variable on y is significant. Generally speaking, it is always desirable to remove the independent variables that are not significant to the response from the regression equation, thus simplifying the regression equation and making it practical. To this end, a significant test of each regression coefficient is required, with reference to the partial regression coefficient test in the multiple regression equation $\mathrm{F}$ test.

\subsection{Prediction and Evaluation of Voltage Spikes Based on Linear Regression Model}

As can be seen from Table 3, since the regression equation and the regression coefficient are all verified, the regression equation can be used to predict the voltage spike. The measured value of the voltage spike and the predicted value are shown in Fig.

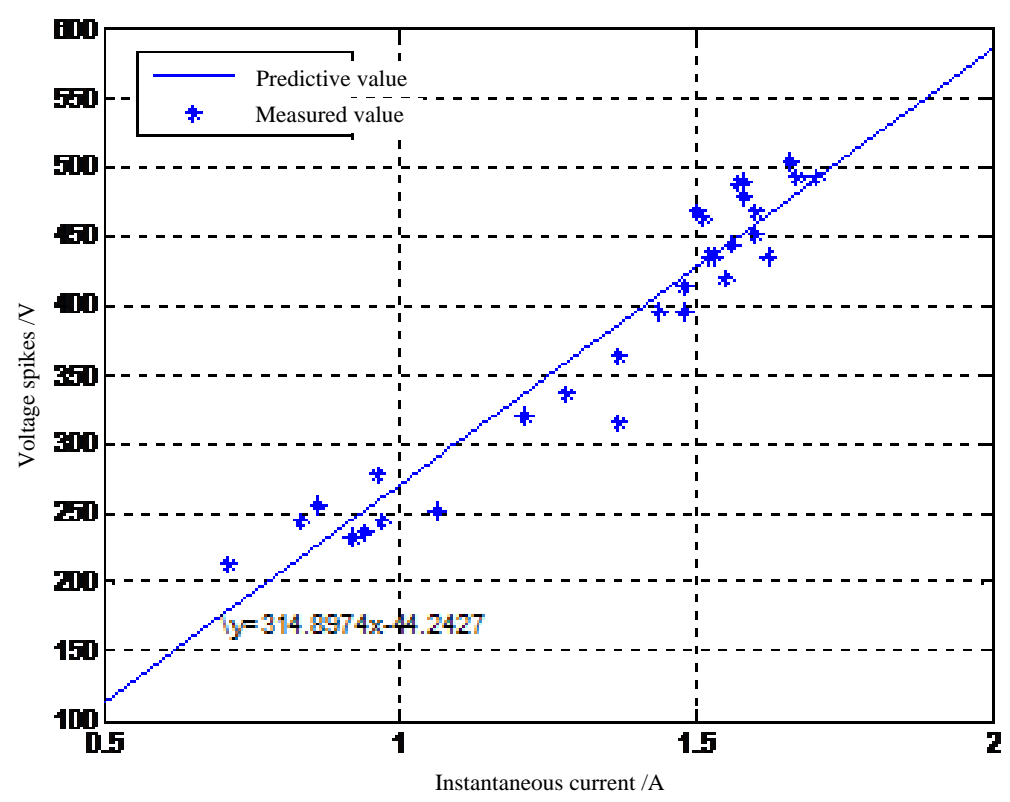

Figure 5. Measured value and predicted trend of voltage spikes

The accuracy of the voltage spike prediction is as follows:

The higher the instantaneous current is, the higher the voltage spike is, so the current peak $\mathrm{x}$ is about 1.70A according to Fig. Conclusion: Using the test results in Table 2 as the statistical sample, the voltage spike prediction based on the linear regression model is $491 \mathrm{~V}$, and the confidence interval is $953 \%(433 \mathrm{~V}, 549 \mathrm{~V})$ when the confidence level is $95 \%$.

\section{Summary}

(1). In the power line voltage spike test, for the inductive load, the voltage spike is mainly affected by the instantaneous current when the switch is turned off, and can be expressed by the linear regression model. The variance analysis of the test results shows that the linear regression characteristics are significant.

(2). The correlation test based on variance analysis shows that the current probe has no significant effect on the voltage spike amplitude test of the power supply line. However, the parallel impedance difference of different types of voltage probes will cause the impedance parameters of the coupling network to be different, so that in the power line voltage spike amplitude test resulting in a more obvious systematic error.

(3). The voltage spike prediction bias based on the linear regression model obeys the normal distribution, which can be used to evaluate the accuracy of the measurement results. 


\section{References}

[1] Moreira A F, Lipo T A, Venkataramanan G, et al. High-frequency modeling for cable and induction motor overvoltage studies in long cable drives[J]. IEEE Transactions on Applications, 2002, 38(5): 1297-1306.

[2] Doyle, Busse F, Jay M E. The effects of PWM voltage source inverter on the mechanical performance of rolling bearings[J] . IEEE Transactions on Industry Applications, 1997, 33(2): 567-576.

[3] Boglietti A, Cavagnino A, Lazzari M. Experimental high frequency parameter identification of AC electrical motors[C] . IEEE International Conference on Electric Machines and Drives, San Antonio, Texas, USA, 2005.

[4] Naik R, Nondhal T A. Circuit model for shaft voltage prediction in induction motors fed by PWM-based AC drives[J]. IEEE Transactions on Industry Applications, 2003, 39(5): 1294-1299. 